\title{
Radiomics \& Radiology: A Critical Step towards Integrated Healthcare
}

\author{
Mayur Pankhania ${ }^{\circledR}$, Aditya Mehta ${ }^{\circledR 2}$ \\ ${ }^{1}$ Senior Consultant Radiologist, Sahyog Imaging Centre, Department of Radiodiagnosis, PDU Medical College \& Government Hospital, Rajkot, Gujarat, India, ${ }^{23 r d}$ year \\ Resident, Department of Radiodiagnosis, PDU Medical College \& Government Hospital, Rajkot, Gujarat, India.
}

\section{Abstract}

Radiomics have shown great promise for integrated healthcare. Radiomics is defined as high-performance retrieval of significant volumes of characteristics from images and conversion of images to higher-dimensional data and subsequently mining for improved support for therapeutic judgements. It has its roots within Computer-Aided Detection (CAD)or Computer-Aided Diagnosis (CADx); it is unique in many aspects. It does not just detect and diagnose but also ventures into therapeutic, prediction, projection and modelling that can be used to generalize and reproduced. It has great potential in creating a paradigm shift in the way healthcare is delivered and perceived. We will review and outline the stage of radiomics\& its SWOT analysis, exclusively addressing application in medical imaging and spotlighting the technical issues.

Keywords: Radiology, Radiomics, Healthcare, CAD

Corresponding Author: Mayur Pankhania, Senior Consultant Radiologist, Sahyog Imaging Centre, Department of Radiodiagnosis, PDU Medical College \& Government Hospital, Rajkot, Gujarat, India.

E-mail: mayurpankhania@gmail.com

Received: 02 June 2020

Revised: 06 July 2020

Accepted: 16 July 2020

Published: 30 December 2020

\section{Introduction}

Medicine is moving from being a reactive discipline to a proactive discipline. We don't just aim to treat diseases now, but we also predict the factors associated with health and disease, promote health, prevent diseases and control their spread. The data-driven, evidence-based solutions form the backbone of today's health care revolution. We are moving away from empirical practices towards genetic and personalized medicine. Information Technology (IT) has galvanized the way the patients interact with the health care providers and the health care systems. Today's healthcare systems are complex with multiple players, and achieving health is a multi-stakeholder endeavour. We are moving away from isolated and unidimensional care to holistic and integrated health care.

Advances in information technology (IT) and computational sciences have given medicine tools to predict, project, and model both physiological and pathological states. Innovations around of using imaging data to predicting actions started in the 1960s. Its systematic application in medicine began in the 1980s. Computer-aided detection and diagnosis (CAD) methods paved the way in this direction for the development of future tools.
While Radiomics has its roots in CAD, it is unique in many aspects. The numeral imaging features that are involved in many folds more significant than that required in CAD. The scope of radiomics is far more extensive than CAD as it does not just detect and diagnose but also ventures into therapeutic, prediction, projection and modelling that can be used to generalize and reproduced. It is a process than a standalone system and helps in both hypothesis generation and testing. It has the capability to act as a biomarker that would contribute towards early detection of ailments.

Radiomics is defined as high-performance retrieval of significant volumes of characteristics from images and conversion of images to higher-dimensional data and subsequently mining for improved support for therapeutic judgement. ${ }^{[1,2]}$ Radiomics is applicable to many biomedical areas though the majority of its application till now is in oncology.

Data from numerous imaging approaches like USG, CT, MRI, PET-CT can be used in the extraction of data for radiomics. Avanzo et al. ${ }^{[3]}$ found that the commonly used imaging modality in radiomics studies is CT. ${ }^{[3]}$ The general lexicon used in radiology is qualitative. The invention of CT \& MRI made the image-based quantitation model feasible from a strictly qualitative view. It exploited the advances in information and technology (IT) and information in the images that reflect the underlying physiology and pathology 
to look beyond what is visible and assists radiologists in arriving at conclusions that are not feasible with an anatomical description alone.

The full collection of imaging features acquired for a patient from the available radiological investigations is called the "radiome". [2] A set of properties that carry prognostic and/or predictive importance are sometimes referred to as a "radiomic signature". Radiomics is based on the hypothesis that quantitative examination of tumours across a wide range of imaging features may provide useful diagnostic, prognostic or predictive information Radiomic signatures, ${ }^{[4]}$ alone or with other patient-specified data, may be employed to enhance diagnosis, prediction of treatment response and prognosis.

\section{The Radiomics Framework}

The operation of radiomics consists of the following steps.

\section{Acquisition \& Restoration of image}

2. Segmentation and interpretation of tumour

3. Extraction of features

4. Predictive modelling

\section{Model Validation}

\section{Acquisition \& Restoration of Image}

Radiomics applies to various images. This provides a large pool of images that can be acquired for radiomic analysis. Increased digitalization of healthcare, the introduction of electronic medical records and the establishment of Hospital Management and Information Systems (HMIS) makes the process more ease. The advent of big data and the evolution of computational methods and statistical methods allow the integration of multiple data sources.

The basic principle in any predictive model is to acquire as much as available data at the front and then use it to recognize the characteristics with the maximum predictive scope. The same is used in radiomics. While as small as hundreds of images can be used to mine the data, availability of large data sets would make the model more valid and comprehensive.

There are some Challenges that can hamper this step. Medical images are acquired in general with the objective of diagnosis and to augmenting the distinction between healthy and pathological tissues. Various hospitals and diagnostic centres are not having uniformity in protocols for image acquisition and are using different parameters for image reconstruction. It could significantly affect the image quality.

\section{Segmentation and Interpretation of Tumour}

Segmentation is a crucial step where the acquired image is segmented to obtain Regions of interest (ROI). The subsequent extraction of features is dependent on the segmentation. Most cruciate and challenging part because many structures like tumour having indistinct borders and also there may be manual contouring variation between operators. In order to account for intra and inter-rater differences, it is essential to assess the reliability of the image characteristics and their effect on further analysis by manipulating the tumour contours or using multiple demarcations. Instead, tumours can be more accurately contoured using semi-automated segmentation algorithms where there is minimal human interference required.

In one of the studies, Kumar et al. noted that automatic segmentations frequently ended in failure using rule-based methods in these situations, as demonstrated by extending the lung borders into the mediastinum or the heart. Furthermore, partly solid ground-glass opacities (GGOs), nodules adjacent to vessels and nodules adjacent to pleural walls are hard to segment automatically and exhibit low reproducibility, especially for Stage III \& Stage IV disorders. ${ }^{[4]}$

\section{Feature Extraction}

The two types of radiomic characteristics that can be derived from the images are semantic and agnostic. Semantic aspects are focused on an established radiology terminology to characterize tumours qualitatively; but at the other end, agnostic characteristics are numerical measures with predetermined mathematical equations. Test-retest data are also immensely beneficial where available because they can better predict features based on their reproducibility.

\section{Predictive Modelling}

After the characteristics are obtained, artificial intelligence or statistical algorithms may be used to check for patterns that correlate with specific clinical endpoints or biological/genomic traits. Image features that relate with a condition can be preferentially selected. Besides building predictive models with supervised algorithms, exploratory unsupervised algorithms can also be used to discover newer features. The unsupervised algorithms provide summary information than the intermediate steps. Radiomics data may contain statistics of the first, second and higher levels. Such data are coupled with other data specific to patients such as backgrounds, ethnicity, physiological, serum markers, molecular clinical or genetic data to obtain predictive, diagnostic or prognostic models more accurate. Model consistency depends not only on the acquired images but also on other coexisting and associated variables. 


\section{Model Validation}

Once the algorithms or models are prepared, validating, it is vital for generalize ability and for prototyping. It should preferably be authenticated on independent multiple external cohorts. It would be synergistic to assess the correlation between the proposed radiomics signatures with the existing clinical \&pathological factors.

\section{Review of the Existing Literature in Radionics}

Several radiomic studies have discovered new imaging signatures that had showed improved diagnostic, prognostic and predictive accuracies than the existing methods. ${ }^{[5]}$

Aert et al. proposed that radiomic characteristics would be used to evaluate the optimal survival of radiotherapytreated patients with pulmonary cancer. To describe the scale, shape and texture of the tumour, more than 400 quantitative properties were derived from $\mathrm{CT}$ images. They developed a radiomic characteristic that obtained intratumor heterogeneity based on these particular parameters and characteristics, which was seen as being of predictive significance in many external validation cohorts, including one head-and-neck cohort. ${ }^{[6]}$

Similarly, radiomics research has been used to examine the association of MRI characteristics with patient outcomes in glioblastoma. ${ }^{[7]} \mathrm{Wu}$ and others. Studied the quantitative radiomic attributes of FDG-PET and $\mathrm{CT}$ for metastatic cancer estimation in early-stage non-small cell lung cancer ( NSCLC) following stereotactic ablative radiotherapy (SABR) ${ }^{[8]} \mathrm{Cui}$ et al. perform a review to classify FDG-PET radiomic characteristics to predict survival levels in 139 advanced pancreatic cancer patients treated with SABR. ${ }^{[9]}$ Van Rossum and others. Investigated if the quantitative assessment of baseline and post-treatment FDG-PET will provide a better predictor of pre-surgical chemoradiotherapy response in oesophageal cancer compared with general patient characteristics. ${ }^{[10]}$ El Naqa and others studied and combined FDG-PET / CT radiomics with medical expertise to assess the risk of recurrence and metastatic cancer of the head and neck. ${ }^{[11]}$ Fehr D employed radiomics technology to identify prostate cancer from healthy prostate tissue and to estimate chances of malignancy. ${ }^{[12]}$ Coroller et al utilized radiomics to determine the response to treatment of lung cancer patients and patients with multiform glioblastoma. ${ }^{[13]}$ Wibmer et al. examined 147 men with prostate cancer and reported that examination of the texture in Haralick would degrade cancer cells and healthy tissue in the prostate. ${ }^{[14]}$ Grove et al. employed radiomics to classify robust prognostic markers in early-stage lung cancer patients. They have also shown a way of delineating healthy tissues inside lesions. ${ }^{[15]}$ In patients who underwent gene expression screening for glioma, Gevaert et al. reported a small number of semanthropic and agnostic properties. ${ }^{[16]}$ Segal et al. contrasted semantine radiologist- identified features from contrast-enhanced CT images in hepatocellular carcinoma patients to recognize patterns of gene expression by using neural network machine learning. ${ }^{[17]}$ Diehn et al. contrasted image characteristics derived from MR images for predicting patterns of global gene expression in patients with multiform glioblastoma. ${ }^{[18]}$ Kuo et al. identified phenotypes for the imaging of hepatocellular carcinoma that correlated with an expression system for the drug response gene doxorubicin. ${ }^{[19]}$ In 49 patients with prostate cancer, Vignati et al. carried out a detailed prospective radiomic study of diffusion- and T2weighted MR imaging tests. ${ }^{[20]}$

Radiomics is also used in clinical treatment as proposed by Teruel et al. In women who sought treatment for breast cancer, it was recommended that texture analysis of dynamic contrast-enhanced MR imaging could help assess the effects to neoadjuvant chemotherapy prior to initiation. ${ }^{[21]}$

Radiomics is used to determine the need for more Investigations as proposed by Klaeser et al., They showed that PET / CT-guided biopsies had a significant clinical effect in patients who otherwise could not be accurately categorized based on traditional assessment. ${ }^{[22]}$ Tatli et al., also performed PET / CT-guided abdominal biopsies using previous PET / CT images obtained with intra-procedural CT scans, to see is the feasibility of using fluorine 18 fluorodeoxyglucose-avid masses that are not properly shown with non-enhanced CT. ${ }^{[23]}$

\section{Strengths of Radiomics in achieving Integrated Healthcare}

- The data required for the radiomics input are taken from the usual standard of care images, and no unique methods of image creation are necessary. With the digitization of healthcare, it is easier to obtain data on health.

- It brings all the levels of healthcare under one umbrella including the promotion of health, prevention of disease, early detection, cure and care and rehabilitation.

- Radiomics can be defined for both populations and individuals and can be used strategically in both.

- As a biomarker, it would synergize the efficacy of drug development and creating new models for clinical trials. These would fast track the availability of diagnostic and therapeutic devices to the patients.

- It has the potential to integrate all dimensions of health, including the physical, mental, emotional, and biological with improved algorithms and would pave the way for holistic health.

- The increases use of radiomics makes the patient care more objective, systematic and personalized. 


\begin{tabular}{|c|c|c|c|c|}
\hline Sl. No & First Author & Image Modality & Organ/Pathology & Outcome \\
\hline 1. & Aerts & CT & $\begin{array}{l}\text { Lung Cancer } \\
\text { \&Glioblastoma Multi- } \\
\text { forme }\end{array}$ & $\begin{array}{l}\text { Survival, Gene Expres- } \\
\text { sion }\end{array}$ \\
\hline 2. & Coroller & CT & Lung Adenocarcinoma & Distant Metastasis \\
\hline 3. & Coroller & CT & NSCLC & $\begin{array}{l}\text { Pathological Response } \\
\text { to neoadjuvant } \\
\text { Chemoradiation }\end{array}$ \\
\hline 4. & Cui & FDG-PET & $\begin{array}{l}\text { Pancreatic Patients } \\
\text { treated with SABR }\end{array}$ & Survival \\
\hline 5. & Diehn & MRI & $\begin{array}{l}\text { Glioblastoma Multi- } \\
\text { form }\end{array}$ & $\begin{array}{l}\text { Global Gene Expres- } \\
\text { sion Pattern }\end{array}$ \\
\hline 6. & El Naqa & FGD-PET & Head \& Neck Cancer & $\begin{array}{l}\text { Loco regional Recur- } \\
\text { rence\& Metastases }\end{array}$ \\
\hline 7. & Fehr D & MRI & Prostate & $\begin{array}{l}\text { Differentiate Benign } \\
\text { from Malignant }\end{array}$ \\
\hline 8. & Gevaert & MRI & $\begin{array}{l}\text { Glioblastoma multi- } \\
\text { forme }\end{array}$ & $\begin{array}{l}\text { Gene Expression Pro- } \\
\text { filing }\end{array}$ \\
\hline 9. & Grossmann & MRI & Glioblastoma & Survival and Prognosis \\
\hline 10. & Grove & $\mathrm{CT}$ & $\begin{array}{l}\text { Early stage Lung Can- } \\
\text { cer }\end{array}$ & Prognosis Indicators \\
\hline 11. & Klaeser & $\mathrm{PET} / \mathrm{CT}$ & Bone Lesions & $\begin{array}{l}\text { Image guidance Biop- } \\
\text { sies }\end{array}$ \\
\hline 12. & Kuo & CECT & $\begin{array}{l}\text { Hepatocellular Carci- } \\
\text { noma }\end{array}$ & $\begin{array}{l}\text { Drug Response gene } \\
\text { expression program }\end{array}$ \\
\hline 13. & Segal & CECT & $\begin{array}{l}\text { Hepatocellular Carci- } \\
\text { noma }\end{array}$ & $\begin{array}{l}\text { Gene Expression Pat- } \\
\text { terns }\end{array}$ \\
\hline 14. & tatli & $\mathrm{PET} / \mathrm{CT}$ & Abdomin & Guidance for Biopsy \\
\hline 15. & Teruel & DCE_MRI & $\begin{array}{l}\text { Locally Advanced } \\
\text { Breast Cancer }\end{array}$ & $\begin{array}{l}\text { Clinical \& Patho- } \\
\text { logical Response to } \\
\text { chemotherapy }\end{array}$ \\
\hline 16. & Van Rossum & FDG-PET & Oesophageal Cancer & Pathological response \\
\hline 17. & Vignati & MRI & Prostate Cancer & $\begin{array}{l}\text { Predict Prostate Can- } \\
\text { cer Aggressiveness }\end{array}$ \\
\hline 18. & Wibmer & T2- weighted MRI & Prostate & $\begin{array}{l}\text { Differentiate Benign } \\
\text { from Malignant }\end{array}$ \\
\hline 19. & $\mathrm{Wu}$ & CT \& FDG-PET & $\begin{array}{l}\text { Pancreatic Patients } \\
\text { treated with SABR }\end{array}$ & Survival \\
\hline
\end{tabular}

\section{Weaknesses of Radiomics in contributing to Integrated Healthcassociations.}

- Radiomic analyses at present are mainly used to identify correlations and not causes. In turn, the definitive assessment is highly unlikely.

- Data mining can work as a double-edged sword. The same features of radiomics that could benefit the patients may also make it redundant with the glorification of spurious
- Radiomics suffers from the same weakness as Artificial Intelligence (AI) and Machine Learning (ML). Some of the layers in unsupervised learning are hidden, and the output created cannot always be evaluated for accuracy.

- Variation is part of human biology and is not always abnormal. This may be overlooked by the radiomics. 


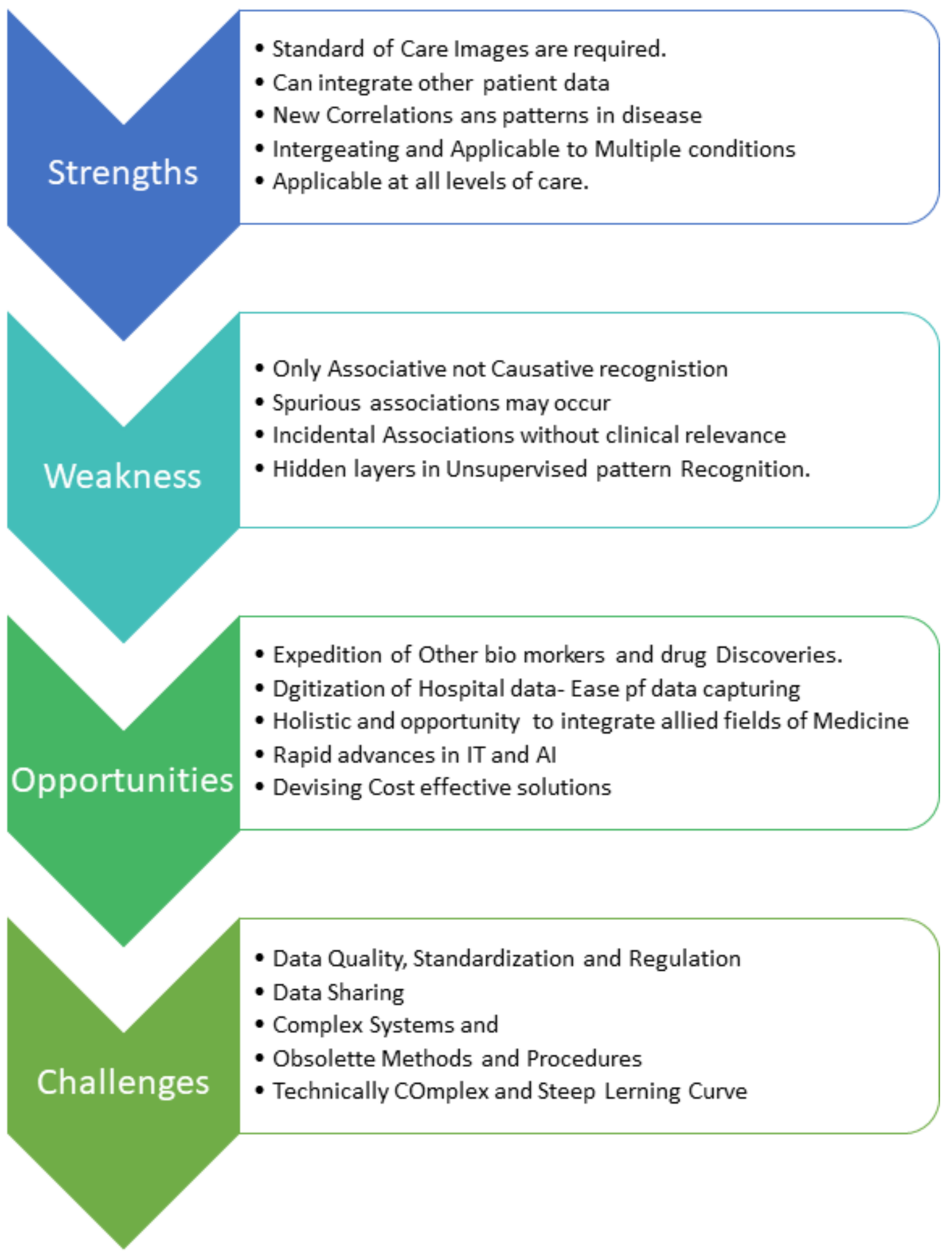


- The incidental associations that would not have any clinical relevance could get highlighted and cause an opportunistic cost in terms of money, human resources and healthcare infrastructure.

\section{Opportunities for the further expansion of Radiomics}

- Radiomic promises to increase diagnostic precision, prognostic assessment and prediction of response to treatment, which are keystones for personalized and precision medicine

- Radiomics can also be a unique opportunity to reach the unreached. The extraction of new signatures would not only strengthen the clinical care but would also a way forward for the robust public health system.

- Advances in radiomics, when made part of routine care would be cost-effective and would provide the health solutions in an economically efficient way.

- It would assist the healthcare providers in decisions and in creating better strategies for patient care.

- Radiomics also can be one of the avenues to unify allopath with allied fields of medicine to provide integrated care.

- - It will have the opportunity for use in care tracking and optimization. Although radiomics techniques are still to be explored in detail, it is a direction worth investigating.

- Throughout the future, the use of radiomics would possibly incorporate image analysis with all the data from the experiments, offering a single source of big data that will expand the potential with discovering useful correlations.

\section{Challenges for Radiomics in achieving the full potential}

- While radiomics will allow patients and their diseases to be better represented, it will also bring more data management challenges. Radiomics is theoretically challenging and has a steep learning curve.

- Wrong research approach, lack of standards for results verification; incomplete reporting of results, and unrecognized perplexing variables in multiple data will introduce model errors and defeat the radiomics intent.

- Lack of standardization of protocols for image capture and retrieval of the attributes. There are still no standards or regulation for standardizing such procedures.

- There is a broad variance in imaging parameters in routine clinical images acquired, like picture quality (pixel size or matrix size and slice thickness), washout time for PET imaging, patient location, and differences introduced by various reconstruction algorithms and slice thicknesses, which are distinct for each vendor of scanners. This may all lead to imprecise predictions.

- The most serious difficulty of all biomedical science is data sharing. It has many obstacles, including financial, economic, regulatory and personal matters. Confidentiality of patient data is another thing to remember when exchanging data.

- Imaging statistics show consistent changes or progress as the acquisition of medical images, and changes in parameters lead to difficulties inconsistent data gathering and some may become outdated before using it.

- Clinical and molecular data integration - the incorporation of data through systems is often a challenge and dynamic in large environments.

\section{Strategies for Way Forward}

The strategies to tackle data sharing is already underway through some of the collaborations like Quantitative Imaging Biomarkers Alliance (QIBA) and the Quantitative Imaging Network (QIN). ${ }^{[24,25]}$ Cancer imaging database projects, where image and clinical records are collected and publicly exchanged for different tumour sites. Pseudo studies can be introduced to examine the interscanning and inter-vendor variation of the features extracted from the imaging. ${ }^{[26,27]}$ Devising better methods for de-identification of patients would help in overcoming the patient data confidentiality issues. Creating an Integrated Radiomics Database would solve the problems of data sharing. Different methods for same can be explored, including the creation of a central repository or, a network of regulated individual databases or creation of a third party for the monitoring database. Appropriate strategies may be decided through discussion and dialogue. Physicians must actively involve in this process, as they will play a crucial role in the whole process. Strengthening the healthcare system by the creation of Information management systems is essential to enhance the entire process. ${ }^{[28]}$

\section{Conclusion}

Radiomics have shown great promise for integrated healthcare. It has great potential in creating a paradigm shift in the way healthcare is delivered and perceived. It has promised to augment the diagnostic precision, prognostic assessment and prediction of response to therapy. While we can be optimistic, there is still a long way before it can become a reality. It needs the contributions and co-operation not only from Radiologist but from multiple stakeholders like data managers, administrators, patients, regulatory bodies and engineers, and who can work in tandem to standardize the methodology and to bring out the full potential of this robust field of radiomics. 


\section{References}

1. Gillies RJ, Kinahan PE, Hricak H. Radiomics: Images Are More than Pictures, They Are Data. Radiology. 2016;278(2):563-577. Available from: https://dx.doi.org/10. 1148/radiol.2015151169.

2. Kotrotsou A, Zinn PO, Colen RR. Radiomics in Brain Tumors: An Emerging Technique for Characterization of Tumor Environment. Magn Reson Imaging Clin N Am . 2016;24(4):719-748. Available from: https://doi.org/10.1016/ j.mric.2016.06.006.

3. Avanzo M, Stancanello J, Naqa IE. Beyond imaging: The promise of radiomics. Phys Med. 2017;38:122-161. Available from: https://doi.org/10.1016/j.ejmp.2017.05.071.

4. Kumar V, Gu Y, Basu S, Berglund A, Eschrich SA, Schabath $\mathrm{MB}$, et al. Radiomics: the process and the challenges. Magn Reson Imaging. 2012;30(9):1234-1248. Available from: https: //dx.doi.org/10.1016/j.mri.2012.06.010.

5. Wu J, Tha KK, Xing L, Li R. Radiomics and radiogenomics for precision radiotherapy. J Radiat Res. 2018;59(1):25-31. Available from: https://dx.doi.org/10.1093/jrr/rrx102.

6. Aerts H, Velazquez ER, Leijenaar R, Parmar C, Grossmann $\mathrm{P}$, Cavalho S. Decoding tumour phenotype by noninvasive imaging using a quantitative radiomics approach. Nat Commun. 2014;5(4006). Available from: https://doi.org/10. 1038 /ncomms5006.

7. Grossmann P, Narayan V, Chang K, Rahman R, Abrey L, Reardon DA, et al. Quantitative imaging biomarkers for risk stratification of patients with recurrent glioblastoma treated with bevacizumab. Neuro-Oncology. 2017;19:1688-1697. Available from: https://dx.doi.org/10.1093/neuonc/nox092.

8. Wu J, Aguilera T, Shultz D, Gudur M, Rubin DL, Loo BW, et al. Early-Stage Non-Small Cell Lung Cancer: Quantitative Imaging Characteristics of $18 \mathrm{~F}$ Fluorodeoxyglucose PET/CT Allow Prediction of Distant Metastasis. Radiology. 2016;281(1):270-278. Available from: https://dx.doi.org/10. 1148/radiol.2016151829.

9. Cui Y, Song J, Pollom E, Alagappan M, Shirato H, Chang DT, et al. Quantitative Analysis of 18F-Fluorodeoxyglucose Positron Emission Tomography Identifies Novel Prognostic Imaging Biomarkers in Locally Advanced Pancreatic Cancer Patients Treated With Stereotactic Body Radiation Therapy. Int J Radiat Oncol Biol Phys. 2016;96(1):102-109. Available from: https://dx.doi.org/10.1016/j.ijrobp.2016.04.034.

10. van Rossum PSN, Fried DV, Zhang L, Hofstetter WL, van Vulpen M, Meijer GJ, et al. The Incremental Value of Subjective and Quantitative Assessment of 18F-FDG PET for the Prediction of Pathologic Complete Response to Preoperative Chemoradiotherapy in Esophageal Cancer. J Nuclear Med. 2016;57(5):691-700. Available from: https://dx. doi.org/10.2967/jnumed.115.163766.

11. Vallières M, Kay-Rivest E, Perrin LJ, Liem X, Furstoss C, Aerts $\mathrm{H}$, et al. Radiomics strategies for risk assessment of tumour failure in head-and-neck cancer. . Sci Rep. 2017;7(1):10117. Available from: https://doi.org/10.1038/s41598-017-10371-5.

12. Fehr D, Veeraraghavan H, Wibmer A, Gondo T, Matsumoto K, Vargas HA, et al. Automatic classification of prostate cancer Gleason scores from multiparametric magnetic resonance images. Proc Natl Acad Sci. 2015;112(46):E6265-E6273. Available from: https://dx.doi.org/10.1073/pnas.1505935112.

13. Coroller TP, Grossmann P, Hou Y, Velazquez ER, Leijenaar RTH, Hermann G, et al. CT-based radiomic signature predicts distant metastasis in lung adenocarcinoma. Radiother Oncol. 2015;114(3):345-350. Available from: https://dx.doi.org/10. 1016/j.radonc.2015.02.015.

14. Wibmer A, Hricak H, Gondo T, Matsumoto K, Veeraraghavan $\mathrm{H}$, Fehr D, et al. Haralick texture analysis of prostate MRI: utility for differentiating non-cancerous prostate from prostate cancer and differentiating prostate cancers with different Gleason scores. Eur Radiol. 2015;25(10):2840-2850. Available from: https://dx.doi.org/10.1007/s00330-015-37018.

15. Gatenby RA, Grove O, Gillies RJ. Quantitative Imaging in Cancer Evolution and Ecology. Radiology. 2013;269(1):8-14. Available from: https://dx.doi.org/10.1148/radiol.13122697.

16. Gevaert O, Mitchell LA, Achrol AS, Xu J, Echegaray S, Steinberg GK, et al. Glioblastoma Multiforme: Exploratory Radiogenomic Analysis by Using Quantitative Image Features. Radiology. 2015;276(1):313-313. Available from: https://dx. doi.org/10.1148/radiol.2015154019.

17. Segal E, Sirlin CB, Ooi C, Adler AS, Gollub J, Chen X, et al. Decoding global gene expression programs in liver cancer by noninvasive imaging. Nat Biotechnol. 2007;25(6):675-680. Available from: https://dx.doi.org/10.1038/nbt1306.

18. Diehn M, Nardini C, Wang DS, McGovern S, Jayaraman M, Liang $\mathrm{Y}$, et al. Identification of noninvasive imaging surrogates for brain tumor gene-expression modules. Proc Natl Acad Sci. 2008;105(13):5213-5218. Available from: https://dx.doi.org/ 10.1073/pnas.0801279105.

19. Kuo MD, Gollub J, Sirlin CB, Ooi C, Chen X. Radiogenomic Analysis to Identify Imaging Phenotypes Associated with Drug Response Gene Expression Programs in Hepatocellular Carcinoma. J Vasc Interv Radiol. 2007;18(7):821-830. Available from: https://dx.doi.org/10.1016/j.jvir.2007.04.031.

20. Vignati A, Mazzetti S, Giannini V, Russo F, Bollito E, Porpiglia F, et al. Texture features on T2-weighted magnetic resonance imaging: new potential biomarkers for prostate cancer aggressiveness. Phys Med Biol. 2015;60(7):26852701. Available from: https://dx.doi.org/10.1088/0031-9155/ $60 / 7 / 2685$.

21. Teruel JR, Heldahl MG, Goa PE, Pickles M, Lundgren S, Bathen TF, et al. Dynamic contrast-enhanced MRI texture analysis for pretreatment prediction of clinical and pathological response to neoadjuvant chemotherapy in patients with locally advanced breast cancer. NMR Biomed. 2014;27(8):887-896. Available from: https://dx.doi.org/10.1002/nbm.3132.

22. Klaeser B, Wiskirchen J, Wartenberg J, Weitzel T, Schmid RA, Mueller MD, et al. PET/CT-guided biopsies of metabolically active bone lesions: applications and clinical impact. Eur J Nucl Med Mol I. 2010;37(11):2027-2036. Available from: https://dx.doi.org/10.1007/s00259-010-1524-z.

23. Tatli S, Gerbaudo VH, Mamede M, Tuncali K, Shyn PB, Silverman SG. Abdominal Masses Sampled at PET/CT-guided Percutaneous Biopsy: Initial Experience with Registration of Prior PET/CT Images. Radiology. 2010;256(1):305-311. Available from: https://dx.doi.org/10.1148/radiol.10090931. 
24. Buckler AJ, Bresolin L, Dunnick NR, and DCS. A Collaborative Enterprise for Multi-Stakeholder Participation in the Advancement of Quantitative Imaging. Radiology. 2011;258(3):906-914. Available from: https://dx.doi.org/10. 1148/radiol.10100799.

25. Kalpathy-Cramer J, Freymann JB, Kirby JS, Kinahan PE, Prior FW. Quantitative Imaging Network: Data Sharing and Competitive AlgorithmValidation Leveraging The Cancer Imaging Archive. Transl Oncol. 2014;7(1):147-152. Available from: https://dx.doi.org/10.1593/tlo.13862.

26. Mackin D, Fave X, Zhang L, Fried D, Yang J, Taylor B, et al. Measuring Computed Tomography Scanner Variability of Radiomics Features. Invest Radiol. 2015;50(11):757-765. Available from: https://dx.doi.org/10. 1097/rli.0000000000000180.

27. Zhao B, Tan Y, Tsai WY, Qi J, Xie C, Lu L, et al. Reproducibility of radiomics for deciphering tumor phenotype with imaging. Sci Rep. 2016;6:23428. Available from: https: //doi.org/10.1038/srep23428.
28. Heilbrun ME. Evaluating RadLex and Real World Radiology Reporting. Acad Radiol. 2013;20:1327-1328. Available from: https://dx.doi.org/10.1016/j.acra.2013.09.011.

Copyright: (C) the author(s), 2020. It is an open-access article distributed under the terms of the Creative Commons Attribution License (CC BY 4.0), which permits authors to retain ownership of the copyright for their content, and allow anyone to download, reuse, reprint, modify, distribute and/or copy the content as long as the original authors and source are cited.

How to cite this article: Pankhania M, Mehta A. Radiomics \& Radiology: A Critical Step towards Integrated Healthcare. Asian J. Med. Radiol. Res. 2020;8(2):23-30.

DOI: dx.doi.org/10.47009/ajmrr.2020.8.2.4

Source of Support: Nil, Conflict of Interest: None declared. 